\title{
Positivity and the attractor dimension in a fourth-order reaction-diffusion equation
}

\author{
By M. V. Bartuccelli ${ }^{1}$, S. A. Gourley ${ }^{1}$ And A. A. Ilyin ${ }^{2}$ \\ ${ }^{1}$ Department of Mathematics and Statistics, University of Surrey, \\ Guildford GU2 7XH, UK (s.gourley@eim.surrey.ac.uk) \\ ${ }^{2}$ Keldysh Institute of Applied Mathematics, 4 Miusskaya Square, \\ Moscow 125047, Russia
}

Received 1 December 2000; revised 18 July 2001; accepted 2 October 2001; published online 22 April 2002

In this paper we investigate the semilinear partial differential equation

$$
u_{t}=-\alpha u_{x x x x}-u_{x x}+u\left(1-u^{2}\right)
$$

with a view, particularly, to obtaining some insight into how one might establish positivity preservation results for equations containing fourth-order spatial derivatives. The maximum principle cannot be applied to such equations. However, progress can be made by employing some very recent 'best possible' interpolation inequalities, due to the third-named author, in which the interpolation constants are both explicitly known and sharp. These are used to estimate the $L^{\infty}$ distance between $u$ and 1 during the evolution. A positivity preservation result can be obtained under certain restrictions on the initial datum. We also establish an explicit two-sided estimate for the fractal dimension of the attractor, which is sharp in terms of the physical parameters.

Keywords: interpolation inequalities; positivity; attractors; fractal dimension

\section{Introduction}

Partial differential equations are frequently used as mathematical models for the evolution of some physical or biological quantity that is positive, such as a population. A realistic mathematical model for such a process should have a positivity-preserving property, i.e. if the solution starts positive, it should stay positive. For example, in many of the mathematical models used in population dynamics, the highest-order spatial derivative term is a Laplacian term. Establishing a positivity preservation result for such a model equation or system is usually a matter of applying the maximum principle (Protter \& Weinberger 1984) or some related result such as invariant set theory for reaction-diffusion systems (Grindrod 1991).

However, the mathematical modelling of some processes involves the use of higherorder spatial derivatives and in such cases the maximum principle cannot be used. In such cases, positivity preservation is extremely difficult to establish and, in fact, may not hold for arbitrary positive initial data. However, one example for which 
positivity preservation is believed to hold is the equation

$$
w_{t}=-w_{x x x x}+\left(\frac{w_{x}^{2}}{w}\right)_{x x}
$$

under periodic boundary conditions, which arises in the study of interface fluctuations and has been considered by Bleher et al. (1994). Actually, these authors were not able to rigorously establish positivity preservation for arbitrary positive initial data, but they did establish a sharp connection between positivity preservation and global existence. They argued that positivity preservation probably does hold, since (1.1) is the scaling limit of a positivity-preserving discrete recursion. Also, they were able to show that solutions do remain positive under various restrictions on the initial data.

Other important contributions to the study of positivity in high-order equations are described in Bernis \& Friedman (1990), Dal Passo et al. (1988) and Bartuccelli et al. $(1998 a, b)$ and the references therein.

In general, fourth-order equations do not exhibit positivity preservation without some restriction on the initial data beyond the requirement that they be positive. And, even then, the establishment of really useful positivity theorems for such equations is a deep and challenging problem. The same applies to proving results on global convergence. In reaction-diffusion equations with only the Laplacian term, comparison theorems are frequently used for this purpose, but these rely on the maximum principle.

In this paper we shall discuss global convergence, positivity and the fractal dimension of the attractor for the initial boundary-value problem

$$
\left.\begin{array}{c}
u_{t}=-\alpha u_{x x x x}-u_{x x}+u\left(1-u^{2}\right), \quad \alpha>0, \quad 0<x<L, \quad t>0, \\
\text { initial condition } u(x, 0)=u_{0}(x), \\
\text { periodic boundary conditions at } x=0, L .
\end{array}\right\}
$$

The partial differential equation in (1.2) is particularly well known in the physics literature, where it is often referred to as the Swift-Hohenberg equation (Swift \& Hohenberg 1977) and sometimes appears in the form

$$
\frac{\partial u}{\partial t}=-\left(1+\frac{\partial^{2}}{\partial x^{2}}\right)^{2} u+\gamma u-u^{3}
$$

with $\gamma>1$. The equilibrium solutions of the Swift-Hohenberg equation correspond to the stationary points of a certain functional arising in studies of second-order materials (see, for example, Coleman et al. 1992; Leizarowitz \& Mizel 1989; Marcus 1993). A similar equation was studied by Pomeau \& Manneville (1980), on a finite one-dimensional spatial domain, as part of a study of wavenumber selection in cellular flows.

Equations with fourth-order derivative terms have also arisen in population dynamics. For example, Cohen \& Murray (1981) derived and studied an equation for a population $n(\boldsymbol{x}, t)$ of the form

$$
\frac{\partial n}{\partial t}=-D k \nabla^{4} n+D A \nabla^{2} n+D B \nabla^{2} n^{3}+G(n),
$$

where $D, B$ and $k$ are positive but $A$ can be of either sign. If $A<0$, this model is similar to ours. In this case, the $D A \nabla^{2} n$ term is destabilizing while the $-D k \nabla^{4} n$ term 
is stabilizing. Cohen \& Murray showed the existence of stationary spatially periodic solutions if $A$ is sufficiently negative. These spatial structures are maintained by the interaction of the short-range 'activation' term $D A \nabla^{2} n$, with $A<0$, versus the long-range inhibition term $-D k \nabla^{4} n$.

There have been a number of papers in the literature dealing with equations similar to the equation in (1.2), but with the $u_{x x}$ term having a positive coefficient. Such equations are sometimes known as extended Fisher-Kolmogorov (EFK) equations and have been proposed as models of phase transitions and other bistable phenomena. Kalies \& van der Vorst (1996) considered the steady-state problem for such an equation, on an infinite spatial domain $x \in(-\infty, \infty)$, and exploited the variational structure of their equation to prove the existence of heteroclinic connections, which are the critical points of a certain functional. Peletier \& Troy (1997) were interested in stationary spatially periodic patterns, showing, in particular, that the structure of the solutions is enriched by increasing the coefficient of the $u_{x x x x}$ term. The structure of the solution set was also investigated by van den Berg (2000), who enumerates all the possible bounded stationary solutions when this coefficient is small. Peletier \& Troy (1998), as a model for travelling waves in suspension bridges, started with the equation $w_{t t}+w_{z z z z}+f(w)=0$, which is the beam equation with a forcing term. Of course, it is second order in time, but a travelling-wave ansatz $w(z, t)=u(x)$, $x=z-c t$ transforms the equation into one like the steady-state problem for (1.2), though with a different nonlinearity. Their interest was in periodic travelling-wave solutions, existence of which they proved by using a topological shooting argument. Periodic and chaotic spatial patterns were investigated by Peletier \& Troy (1996) and Kalies et al. (2000).

Returning to problem (1.2), our main interest in the present paper is in positivity of solutions. The main ideas to be described should also be applicable to other equations with high-order spatial derivatives and polynomial nonlinearities. For example, in Bartuccelli et al. $(1998 a, b)$, some progress is made, but in these papers positivity is only established for sufficiently large times $t$, leaving open the possibility that the solution may go negative during the transients. In the present paper our main positivity result is for all times $t$.

The paper is organized as follows. Section 2 establishes a convergence result, namely, that under a certain condition on the initial data $u(x, 0)$, the solution of $(1.2)$ tends to 1 uniformly in $x$. In $\S 3$ we address positivity. Our main theorem here (theorem 3.1) establishes positivity of the solution of (1.2) for all time, again under certain restrictions on the initial data and on the parameter $\alpha$ and the domain length $L$. Then, in $\S 4$, we derive a two-sided estimate for the fractal dimension of the global attractor. The estimate is given in theorem 4.1. Some concluding remarks are given in $\S 5$.

Our strategy for studying positivity involves centring the equation on the uniform steady-state solution $u \equiv 1$ and then showing that, under some restrictions on the initial data and the parameters $\alpha$ and $L$, solutions of the transformed equation are bounded, in absolute value, by 1 . To show this, we use ideas of energy methods together with some very recent interpolation inequalities due to Ilyin (1998), in which the interpolation constants are explicitly known and are the best possible. This strategy can also be employed to show (under certain other conditions) that solutions of (1.2) converge to $u=1$, uniformly for $x \in[0, L]$.

Proc. R. Soc. Lond. A (2002) 


\section{Convergence}

In this section we establish a convergence result for (1.2). A linearized analysis shows that the $u=1$ solution of (1.2) is unstable when $\alpha<\frac{1}{8}$ and the domain length $L$ is sufficiently large. For (nonlinear) stability of $u=1$, the condition on $\alpha$ that emerges from our analysis is $\alpha>\frac{1}{4}$, as we shall show. We introduce $v(x, t)$, defined by

$$
u(x, t)=1+v(x, t),
$$

where $u$ satisfies (1.2). Also, we denote

$$
\|v(\cdot, t)\|_{\infty}=\sup _{x \in[0, L]}|v(x, t)| .
$$

Showing that $\|v(\cdot, t)\|_{\infty} \rightarrow 0$ as $t \rightarrow \infty$ establishes uniform convergence of solutions of (1.2) to the equilibrium solution $u=1$.

Furthermore, if $v(x, t)$ satisfies

$$
\|v(\cdot, t)\|_{\infty} \leqslant 1 \text { for all } t
$$

then $u(\cdot, t)$ is a non-negative function for all $t$. This will be done, under certain conditions, in $\S 3$.

We introduce the time-dependent quantities

$$
J_{n}:=\left\|\frac{\partial^{n} v}{\partial x^{n}}\right\|_{2}^{2}=\int_{0}^{L}\left(\frac{\partial^{n} v}{\partial x^{n}}\right)^{2} \mathrm{~d} x,
$$

where $v$ denotes the function defined by (2.1).

Substituting (2.1) into (1.2), we obtain for $v$ the equation

$$
v_{t}=-\alpha v_{x x x x}-v_{x x}-2 v-3 v^{2}-v^{3} .
$$

Our aim is now to find conditions that will ensure that $\|v\|_{\infty} \rightarrow 0$ as $t \rightarrow \infty$, which establishes uniform convergence of solutions of (1.2) to the $u=1$ solution thereof.

We first obtain appropriate estimates for the $L^{2}$-norm of the solution of (2.4) and also of its derivative $\partial v / \partial x$. Using these estimates, together with a new interpolation inequality, we then estimate $\|v\|_{\infty}$ in a way that only involves the parameters of our equation and an interpolation constant whose value is explicitly known.

We start our analysis by investigating the evolution of the $L^{2}$-norm of the solution $v$ of (2.4), namely $J_{0}$. Differentiating $J_{0}$ with respect to time and inserting the righthand side of (2.4) gives

$$
\frac{1}{2} \dot{J}_{0}=-\alpha J_{2}+J_{1}-2 J_{0}-3 \int_{0}^{L} v^{3} \mathrm{~d} x-\int_{0}^{L} v^{4} \mathrm{~d} x
$$

where the dot denotes differentiation with respect to time. The $-\alpha J_{2}$ and the $J_{1}$ terms have been obtained by integration by parts (notice that the integrated terms vanish since the boundary conditions are periodic).

By using

$$
J_{1} \leqslant J_{2}^{1 / 2} J_{0}^{1 / 2} \leqslant \frac{1}{2} \alpha J_{2}+\frac{1}{2 \alpha} J_{0}
$$

and

$$
-3 \int_{0}^{L} v^{3} \mathrm{~d} x \leqslant 3\|v\|_{\infty} \int_{0}^{L} v^{2} \mathrm{~d} x=3\|v\|_{\infty} J_{0},
$$

Proc. R. Soc. Lond. A (2002) 


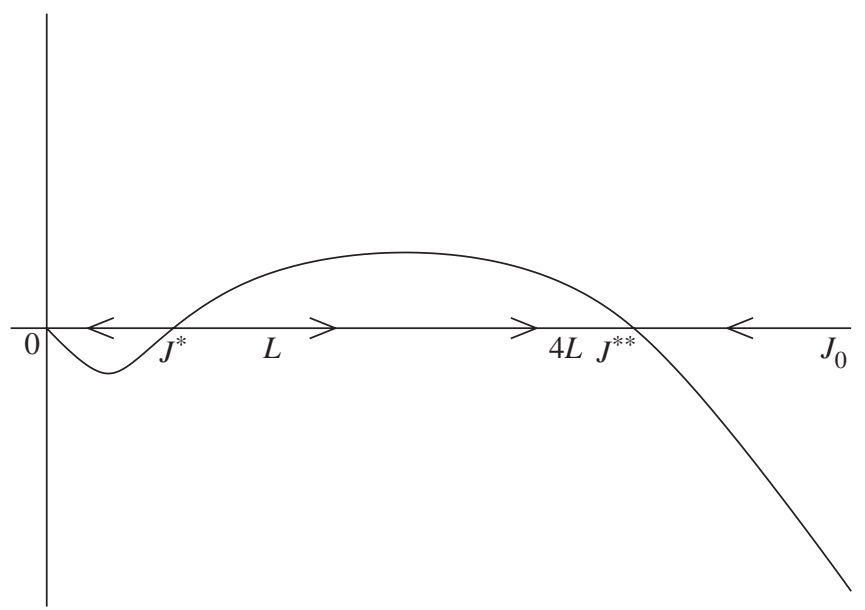

Figure 1. Qualitative sketch of the graph of $f\left(J_{0}\right)$ against $J_{0}$, showing also the behaviour of the solutions of the ODE $\dot{J}_{0}=2 f\left(J_{0}\right)$ for various initial data.

equation (2.5) becomes

$$
\frac{1}{2} \dot{J}_{0} \leqslant-\frac{1}{2} \alpha J_{2}-\left(2-\frac{1}{2 \alpha}\right) J_{0}+3\|v\|_{\infty} J_{0}-\int_{0}^{L} v^{4} \mathrm{~d} x .
$$

Next, the term $\|v\|_{\infty}$ has to be estimated. To do this, we shall use the following sharp interpolation inequality, which is discussed in the appendix (see (A 4), with $l=2)$ :

$$
\|v\|_{\infty} \leqslant c J_{2}(v)^{1 / 8} J_{0}(v)^{3 / 8}+L^{-1 / 2} J_{0}(v)^{1 / 2}, \quad c=\left(\frac{4}{27}\right)^{1 / 8} .
$$

Also, by the Cauchy-Schwarz inequality,

$$
-\int_{0}^{L} v^{4} \mathrm{~d} x \leqslant-J_{0}^{2} L^{-1}
$$

Using these estimates in (2.6) yields

$$
\frac{1}{2} \dot{J}_{0} \leqslant-\frac{1}{2} \alpha J_{2}-\left(2-\frac{1}{2 \alpha}\right) J_{0}+3 c J_{2}^{1 / 8} J_{0}^{11 / 8}+3 L^{-1 / 2} J_{0}^{3 / 2}-J_{0}^{2} L^{-1} .
$$

Next, we use Young's inequality to estimate $3 c J_{2}^{1 / 8} J_{0}^{11 / 8}$ as follows,

$$
3 c J_{2}^{1 / 8} J_{0}^{11 / 8}=\alpha^{1 / 8} J_{2}^{1 / 8} \frac{3 c}{\alpha^{1 / 8}} J_{0}^{11 / 8} \leqslant \frac{1}{8} \alpha J_{2}+\frac{7}{8} \frac{(3 c)^{8 / 7}}{\alpha^{1 / 7}} J_{0}^{11 / 7},
$$

so

$$
\begin{aligned}
\frac{1}{2} \dot{J}_{0} & \leqslant-\frac{3}{8} \alpha J_{2}-\left(2-\frac{1}{2 \alpha}\right) J_{0}+\frac{7}{8} \frac{(3 c)^{8 / 7}}{\alpha^{1 / 7}} J_{0}^{11 / 7}+3 L^{-1 / 2} J_{0}^{3 / 2}-J_{0}^{2} L^{-1} \\
& \leqslant-\left(2-\frac{1}{2 \alpha}\right) J_{0}+\frac{7}{8} \frac{(3 c)^{8 / 7}}{\alpha^{1 / 7}} J_{0}^{11 / 7}+3 L^{-1 / 2} J_{0}^{3 / 2}-J_{0}^{2} L^{-1} \\
& :=f\left(J_{0}\right) .
\end{aligned}
$$


We need to study the solutions of the above differential inequality. By elementary theory, they are bounded above by the solutions of the one-dimensional ordinary differential equation (ODE) $\dot{J}_{0}=2 f\left(J_{0}\right)$. Note that the solutions of (2.4) certainly cannot all satisfy $v \rightarrow 0$ as $t \rightarrow \infty$, since this equation has uniform steady-states $v=-1$ and $v=-2$ in addition to $v=0$. These other states correspond to the uniform steady-states $u=0$ and $u=-1$ of (1.2). At these states, we have $J_{0}=L$ and $J_{0}=4 L$, respectively, and it is easily checked that $f$ is positive at these values. In fact, provided $\alpha>\frac{1}{4}$, the function $f$ is negative for $J_{0}$ small, and for $J_{0}$ large, and positive in some intermediate range. A sketch of its graph is given in figure 1, in which we have also depicted the behaviour of the solutions of the ODE $\dot{J}_{0}=2 f\left(J_{0}\right)$ for various initial data. We may therefore state that if $J_{0}(t=0)<J^{*}$, where $J^{*}$ is the smallest positive root of $f(J)=0$, then $J_{0} \rightarrow 0$ as $t \rightarrow \infty$. Sufficient conditions to ensure this are that $J_{0}(t=0)<1$ and

$$
2-\frac{1}{2 \alpha}>\frac{7}{8} \frac{(3 c)^{8 / 7}}{\alpha^{1 / 7}}\left(J_{0}(t=0)\right)^{4 / 7}+3 L^{-1 / 2}\left(J_{0}(t=0)\right)^{1 / 2} .
$$

Our aim is to establish an estimate for $\|v\|_{\infty}$, with a view to proving that it tends to zero under some conditions. Estimating $\|v\|_{\infty}$ will be achieved by employing a certain inequality that will necessitate our having an estimate on $J_{1}$, where

$$
J_{1}=\int_{0}^{L}\left(\frac{\partial v}{\partial x}\right)^{2} \mathrm{~d} x
$$

It is easily seen that the evolution equation for $J_{1}$ is

$$
\frac{1}{2} \dot{J}_{1}=-\alpha J_{3}+J_{2}-2 J_{1}-6 \int_{0}^{L} v v_{x}^{2} \mathrm{~d} x-3 \int_{0}^{L} v^{2} v_{x}^{2} \mathrm{~d} x
$$

and, after some manipulation, this becomes

$$
\frac{1}{2} \dot{J}_{1} \leqslant-\frac{1}{2} \alpha J_{3}-\left(2-\frac{1}{2 \alpha}\right) J_{1}-6 \int_{0}^{L} v v_{x}^{2} \mathrm{~d} x-3 \int_{0}^{L} v^{2} v_{x}^{2} \mathrm{~d} x .
$$

Using the Cauchy-Schwarz inequality and then Young's inequality, we have

$$
-6 \int_{0}^{L} v v_{x}^{2} \mathrm{~d} x \leqslant\left(6 \int_{0}^{L} v^{2} v_{x}^{2} \mathrm{~d} x\right)^{1 / 2}\left(6 J_{1}\right)^{1 / 2} \leqslant 3 \int_{0}^{L} v^{2} v_{x}^{2} \mathrm{~d} x+3 J_{1},
$$

thus

$$
\frac{1}{2} \dot{J}_{1} \leqslant-\frac{1}{2} \alpha J_{3}+\left(1+\frac{1}{2 \alpha}\right) J_{1} .
$$

By using the inequality $-J_{3} \leqslant-J_{1}^{3} / J_{0}^{2}$ (see Bartuccelli et al. 1993), we have

$$
\frac{1}{2} \dot{J}_{1} \leqslant-\frac{1}{2} \alpha \frac{J_{1}^{3}}{J_{0}^{2}}+\left(1+\frac{1}{2 \alpha}\right) J_{1} .
$$

Taking into account that, under the previously stated conditions, $J_{0} \rightarrow 0$ as $t \rightarrow \infty$, we find from (2.14) that

$$
J_{1}(t) \leqslant \text { const. for all } t \geqslant 0 \text {. }
$$

Proc. R. Soc. Lond. A (2002) 
From the appendix (see (A 4), with $l=1$ ), we have that

$$
\|v\|_{\infty} \leqslant J_{1}^{1 / 4} J_{0}^{1 / 4}+L^{-1 / 2} J_{0}^{1 / 2} \rightarrow 0 \quad \text { as } t \rightarrow \infty
$$

and therefore, from $(2.1)$, that $\lim _{t \rightarrow \infty} u(x, t)=1$, uniformly in $x$.

We may summarize our findings in the following theorem, which gives a condition on the initial conditions that is sufficient for convergence. Of course, we have formulated this theorem in terms of the original state variable $u$. The existence of $J^{*}$ is guaranteed by earlier remarks.

Theorem 2.1. If $\alpha>\frac{1}{4}$ and the initial data satisfy

$$
\int_{0}^{L}(u(x, 0)-1)^{2} \mathrm{~d} x<J^{*}
$$

where $J^{*}$ is the smallest positive root of

$$
f(J):=-\left(2-\frac{1}{2 \alpha}\right) J+\frac{7}{8} \frac{(3 c)^{8 / 7}}{\alpha^{1 / 7}} J^{11 / 7}+3 L^{-1 / 2} J^{3 / 2}-J^{2} L^{-1}=0,
$$

and $c=\left(\frac{4}{27}\right)^{1 / 8}$, then the solution $u(x, t)$ of $(1.2)$ satisfies

$$
\lim _{t \rightarrow \infty} u(x, t)=1
$$

uniformly for $x \in[0, L]$.

Corollary 2.2. If the initial data satisfy

$$
\int_{0}^{L}(u(x, 0)-1)^{2} \mathrm{~d} x<1
$$

and

$$
2-\frac{1}{2 \alpha}>\frac{7}{8} \frac{(3 c)^{8 / 7}}{\alpha^{1 / 7}}\left(\int_{0}^{L}(u(x, 0)-1)^{2} \mathrm{~d} x\right)^{4 / 7}+3 L^{-1 / 2}\left(\int_{0}^{L}(u(x, 0)-1)^{2} \mathrm{~d} x\right)^{1 / 2},
$$

then the same conclusion holds.

\section{Positivity of solutions}

We have shown (theorem 2.1) that, under certain restrictions on the $L^{2}$-norm of the initial data, the solution of (1.2) converges uniformly to 1 . The fact that the convergence is uniform enables us to further deduce that the solution must be positive for all $t$ sufficiently large, but not necessarily for all $t$. We now wish to address the question of positivity for all time.

In the previous section our strategy for proving convergence of the solution to 1 was to show that

$$
\|u(\cdot, t)-1\|_{\infty} \rightarrow 0 \quad \text { as } t \rightarrow \infty
$$

and this was achieved by estimating $\|u(\cdot, t)-1\|_{\infty}$ using the best-known interpolation inequalities available to date. By refining this strategy further, our aim in this section 
is to show that, under certain other restrictions on the initial data, one can establish that, for all values of $t$ (not just as $t \rightarrow \infty$ ),

$$
\|u(\cdot, t)-1\|_{\infty} \leqslant 1 .
$$

If we can establish the above inequality, then we have that $u(x, t) \geqslant 0$ for all $x$ and $t$ and, therefore, that we have positivity preservation. This strategy can be applied in principle to any other partial differential equation.

Some of the hypotheses of theorem 2.1 will still be needed, although we can relax some of the strict inequalities. We assume that $\alpha>\frac{1}{4}$ and that

$$
J_{0}(t=0)=\int_{0}^{L}(v(x, 0))^{2} \mathrm{~d} x \leqslant J^{*},
$$

where $J^{*}$ is as previously defined, and as labelled in figure 1. Under this assumption, we have that

$$
J_{0}(t) \leqslant J^{*} \text { for all } t \geqslant 0 .
$$

We shall use this estimate in (2.14) to deduce that

$$
\frac{1}{2} \dot{J}_{1} \leqslant-\frac{1}{2} \alpha \frac{J_{1}^{3}}{J^{*^{2}}}+\left(1+\frac{1}{2 \alpha}\right) J_{1}
$$

which is an autonomous differential inequality, solutions of which will be bounded above by solutions of the corresponding differential equation. In particular, it is easily seen that if

$$
J_{1}(t=0) \leqslant J^{*} \sqrt{\frac{2}{\alpha}+\frac{1}{\alpha^{2}}}
$$

then

$$
J_{1}(t) \leqslant J^{*} \sqrt{\frac{2}{\alpha}+\frac{1}{\alpha^{2}}} \text { for all } t \geqslant 0 .
$$

Recall that $u-1=v$ and that we have the interpolation inequality

$$
\|v(\cdot, t)\|_{\infty} \leqslant J_{1}^{1 / 4} J_{0}^{1 / 4}+L^{-1 / 2} J_{0}^{1 / 2},
$$

so it is sufficient to show that the right-hand side of (3.4) is bounded above by 1 for all $t \geqslant 0$. In view of the bounds we have for $J_{0}$ and $J_{1}$, given by (3.1) and (3.3), respectively, it is therefore sufficient to show that

$$
\left(J^{*}\right)^{1 / 4}\left(\frac{2}{\alpha}+\frac{1}{\alpha^{2}}\right)^{1 / 8}\left(J^{*}\right)^{1 / 4}+L^{-1 / 2}\left(J^{*}\right)^{1 / 2} \leqslant 1
$$

or, equivalently, that

$$
J^{*} \leqslant \frac{1}{\left(\left(2 / \alpha+1 / \alpha^{2}\right)^{1 / 8}+L^{-1 / 2}\right)^{2}} .
$$

Recall that $J^{*}$ is defined as the smallest positive root of $f(J)=0$, where $f(J)$ is the function defined in the statement of theorem 2.1. It is not immediately obvious that inequality (3.5) can be satisfied for any parameter combination. But, because of the properties of $f(J)$ (see figure 1), the inequality will certainly be satisfied if

$$
f\left(\frac{1}{\left(\left(2 / \alpha+1 / \alpha^{2}\right)^{1 / 8}+L^{-1 / 2}\right)^{2}}\right) \geqslant 0,
$$

Proc. R. Soc. Lond. A (2002) 
and this is equivalent to

$$
G(\alpha, L) \geqslant 0
$$

where

$$
\begin{aligned}
G(\alpha, L)=- & \frac{(2-1 / 2 \alpha)}{\left(\left(2 / \alpha+1 / \alpha^{2}\right)^{1 / 8}+L^{-1 / 2}\right)^{2}}+\frac{7(3 c)^{8 / 7}}{8 \alpha^{1 / 7}\left(\left(2 / \alpha+1 / \alpha^{2}\right)^{1 / 8}+L^{-1 / 2}\right)^{22 / 7}} \\
& +\frac{3}{\sqrt{L}\left(\left(2 / \alpha+1 / \alpha^{2}\right)^{1 / 8}+L^{-1 / 2}\right)^{3}}-\frac{1}{L\left(\left(2 / \alpha+1 / \alpha^{2}\right)^{1 / 8}+L^{-1 / 2}\right)^{4}}
\end{aligned}
$$

and $c=\left(\frac{4}{27}\right)^{1 / 8}$. Extensive numerical investigations demonstrate that for any fixed value of $L, G(\alpha, L)$ is positive until $\alpha$ is extremely large and is then negative and tends to zero from below as $\alpha \rightarrow \infty$. Some asymptotic analysis confirms this; we can show that as $\alpha \rightarrow \infty$,

$$
G(\alpha, L) \sim \frac{1}{\alpha^{1 / 8}}\left(-2^{1 / 8} L^{3 / 2}+\frac{7}{648} 9^{6 / 7}\left(4^{1 / 8} 27^{7 / 8}\right)^{8 / 7} L^{11 / 7} \frac{1}{\alpha^{1 / 56}}\right) .
$$

Of course, we always have to assume that $\alpha>\frac{1}{4}$. Therefore, what we have to assure ourselves of is that, for any given reasonable $L$, there will always be a large range of values of $\alpha$ for which $G(\alpha, L) \geqslant 0$. This is certainly the case. For example, if $L=1$, either a numerical study of the equation $G(\alpha, L)=0$, or its asymptotic approximation as $\alpha \rightarrow \infty$, predicts that the value of $\alpha$ at which $G(\alpha, L)>0$ ceases to hold is of the order of $10^{18}$.

We can now summarize our findings in the following theorem, formulated in terms of the original state variable $u$.

Theorem 3.1. Let $\alpha>\frac{1}{4}$ and let the initial data satisfy

$$
\int_{0}^{L}(u(x, 0)-1)^{2} \mathrm{~d} x \leqslant J^{*},
$$

where $J^{*}$ is the smallest positive root of $f(J)=0$, where $f(J)$ is as in the statement of theorem 2.1. Assume that the initial data also satisfy

$$
\int_{0}^{L}\left(\frac{\partial u}{\partial x}(x, 0)\right)^{2} \mathrm{~d} x \leqslant J^{*} \sqrt{\frac{2}{\alpha}+\frac{1}{\alpha^{2}}}
$$

and assume that $\alpha$ and $L$ are such that

$$
J^{*} \leqslant \frac{1}{\left(\left(2 / \alpha+1 / \alpha^{2}\right)^{1 / 8}+L^{-1 / 2}\right)^{2}} .
$$

Then the solution $u(x, t)$ of (1.2) satisfies $u(x, t) \geqslant 0$ for all $t \geqslant 0$ and all $x \in[0, L]$.

Remark 3.2. One could inquire whether another positivity-preservation theorem could be established by following through the above methodology but using $J^{* *}$, the larger of the two positive roots of $f(J)=0$, rather than $J^{*}$. This leads us to an inequality like (3.5), but with $J^{* *}$ in place of $J^{*}$. But, unfortunately, the resulting inequality is not satisfied by any parameter combination. 


\section{Attractors and their dimension}

It will be convenient for us to rewrite the equation in (1.2) as

$$
u_{t}=-\alpha u_{x x x x}-\beta u_{x x}+\lambda u-u^{3}, \quad u(0)=u_{0} .
$$

Following Babin \& Vishik (1989, §I.5), we can easily show that for each $u_{0} \in H$, where $H=L_{2}(0, L)$, and $T>0$, problem (4.1) has a unique solution $u \in C([0, T] ; H)$ and the corresponding semigroup $S_{t} u_{0}=u(t)$ has a global attractor $\mathcal{A} \subset \subset H$. In this section we derive a two-sided estimate for the fractal dimension of it (see the necessary definitions in Babin \& Vishik (1989), Temam (1997) and Chepyzhov \& Ilyin (2001)). See also Constantin \& Foias (1985) and Constantin et al. (1988), where attractor dimensions are computed in other contexts.

It can be shown (see Babin \& Vishik 1989) that the semigroup $S_{t} u$ is uniformly (quasi) differentiable on $\mathcal{A}$ in $H$ and the differential is the solution operator of the first variation equation

$$
U_{t}=-\alpha U_{x x x x}-\beta U_{x x}+\lambda U-3 u(t)^{2} U \equiv \mathcal{L}(u(t)) \cdot U
$$

corresponding to (4.1). Here, $u(t)=S_{t} u_{0}$.

We recall from Temam (1997) that the sums of the first $m$ global Lyapunov exponents (which we denote by $q(m)$ ) for the attractor $\mathcal{A}$ satisfy the estimate

$$
q(m) \leqslant \limsup _{t \rightarrow \infty} \sup _{u_{0} \in X} \sup _{\substack{\xi_{i} \in H \\ i=1, \ldots m}}\left(\frac{1}{t} \int_{0}^{t} \operatorname{Tr} \mathcal{L}\left(S_{\tau} u_{0}\right) \circ Q_{m}(\tau) \mathrm{d} \tau\right),
$$

where $Q_{m}(t)$ is the orthogonal projection in $H$ onto $\operatorname{Span}\left(U_{1}(\tau), \ldots, U_{m}(\tau)\right)$. Here, the $U_{j}$ are the solutions of problem (4.2) with $U_{j}(0)=\xi_{j}$.

Therefore, we have

$$
\begin{aligned}
\operatorname{Tr} \mathcal{L}\left(S_{t} u_{0}\right) \circ Q_{m}(t)=- & \alpha \sum_{i=1}^{m}\left(\Delta^{2} \varphi_{i}, \varphi_{i}\right)-\beta \sum_{i=1}^{m}\left(\Delta \varphi_{i}, \varphi_{i}\right) \\
& +\lambda \sum_{i=1}^{m}\left(\varphi_{i}, \varphi_{i}\right)-3 u(t)^{2} \sum_{i=1}^{m}\left(\varphi_{i}, \varphi_{i}\right) \\
\leqslant & -\alpha \sum_{i=1}^{m}\left\|\Delta \varphi_{i}\right\|^{2}+\beta \sum_{i=1}^{m}\left\|\nabla \varphi_{i}\right\|^{2}+\lambda m,
\end{aligned}
$$

where the $\varphi_{i}=\varphi_{i}(t)$ are an orthonormal basis in $\operatorname{Span}\left(U_{1}(t), \ldots, U_{m}(t)\right)$.

Since

$$
\left\|\nabla \varphi_{i}\right\|^{2} \leqslant\left\|\varphi_{i}\right\|\left\|\Delta \varphi_{i}\right\| \leqslant \varepsilon\left\|\Delta \varphi_{i}\right\|^{2}+(4 \varepsilon)^{-1}\left\|\varphi_{i}\right\|^{2},
$$

we set $\varepsilon=\alpha /(2 \beta)$ and find that

$$
\operatorname{Tr} \mathcal{L}\left(S_{t} u_{0}\right) \circ Q_{m}(t) \leqslant \frac{1}{2} \alpha \sum_{i=1}^{m}\left\|\Delta \varphi_{i}\right\|^{2}+\left(\frac{\beta^{2}}{2 \alpha}+\lambda\right) m .
$$

Next, in view of the variational principle (see Temam 1997, lemma VI.2.1),

$$
\sum_{i=1}^{m}\left\|\Delta \varphi_{i}\right\|^{2} \geqslant \sum_{i=1}^{m} \lambda_{i}
$$

Proc. R. Soc. Lond. A (2002) 
where the $\lambda_{i}$ are the eigenvalues of the operator $\Delta^{2}$ in $H$ written in non-decreasing order. Obviously,

$$
\left\{\lambda_{i}\right\}_{i=1}^{\infty}=0 \cup\left\{(2 \pi / L)^{4} i^{4},(2 \pi / L)^{4} i^{4}\right\}_{i=1}^{\infty} .
$$

Therefore, if we write $m-1$ as $m-1=2 k+p$, where $p=0,1$, we find that

$$
\begin{aligned}
\sum_{i=1}^{m} \lambda_{i} & =\left(\frac{2 \pi}{L}\right)^{4}\left(p \sum_{i=1}^{k+1} i^{4}+(2-p) \sum_{i=1}^{k} i^{4}\right) \\
& \geqslant\left(\frac{2 \pi}{L}\right)^{4} \frac{1}{5}\left(p(k+1)^{5}+(2-p) k^{5}\right) \\
& =\left(\frac{2 \pi}{L}\right)^{4} \frac{2}{5}\left(\frac{1}{2} p(k+1)^{5}+\frac{1}{2}(2-p) k^{5}\right) \\
& \geqslant\left(\frac{2 \pi}{L}\right)^{4} \frac{2}{5}\left(k+\frac{1}{2} p\right)^{5} \\
& =\left(\frac{\pi}{L}\right)^{4} \frac{1}{5}(m-1)^{5},
\end{aligned}
$$

where we used the inequality $\sum_{i=1}^{k} i^{4} \geqslant \frac{1}{5} k^{5}$ and the fact that the function $k \rightarrow k^{5}$ is convex.

Hence the numbers $q(m)$ (see $(4.3))$ satisfy the following estimate:

$$
q(m) \leqslant g(m)=-\frac{1}{10} \alpha\left(\frac{\pi}{L}\right)^{4}(m-1)^{5}+\left(\frac{\beta^{2}}{2 \alpha}+\lambda\right) m .
$$

The function $g(m)$ is concave. Therefore, in view of the estimate of the fractal dimension established in Chepyzhov \& Ilyin (2001), we have

$$
\operatorname{dim}_{F} \mathcal{A} \leqslant m_{0},
$$

where $m_{0}$ is the unique root of the equation $g(m)=0$. (The condition that $g(m)$ be concave is not necessary (see Ilyin \& Chepyzhov 2000).) However, the root of the equation

$$
-(m-1)^{5}+C m=0, \quad C \gg 1,
$$

has the following asymptotics,

$$
m_{0}=C^{1 / 4}+\frac{5}{4}+\ldots,
$$

and for all $C>0$ we have $m_{0}<C^{1 / 4}+\frac{5}{4}$. Therefore, by (4.8),

$$
\operatorname{dim}_{F} \mathcal{A} \leqslant 10^{1 / 4} \frac{L}{\pi}\left(\frac{\beta^{2}}{2 \alpha^{2}}+\frac{\lambda}{\alpha}\right)^{1 / 4}+\frac{5}{4} .
$$

We now give a lower bound for the dimension of the attractor. We consider the stationary solution $u=0$ and the corresponding linearized operator

$$
A u=-\alpha u_{x x x x}-\beta u_{x x}+\lambda u .
$$

Proc. R. Soc. Lond. A (2002) 
We shall find the number of unstable (positive) eigenvalues of $A$. Then this number is equal to the dimension of the unstable manifold of equation (4.1) passing through $u=0$. Since this unstable manifold is contained in the attractor (Babin \& Vishik $1989, \S$ VI.4), we obtain the required lower bound. Since the spectrum of the operator $A$ is known explicitly (see (4.6)), we see that the dimension of the unstable manifold is $2 K+1$, where $K$ is the number of positive integers $k$ satisfying the inequality

$$
-\alpha\left(\frac{2 \pi}{L}\right)^{4} k^{4}+\beta\left(\frac{2 \pi}{L}\right)^{2} k^{2}+\lambda>0 .
$$

Therefore,

$$
2 K+1=2\left[\frac{L}{2 \pi}\left(\frac{\beta}{2 \alpha}+\sqrt{\frac{\beta^{2}}{4 \alpha^{2}}+\frac{\lambda}{\alpha}}\right)^{1 / 2}\right]+1,
$$

where $[x]$ is the number of positive integers strictly less than $x$.

Combining (4.9) and (4.12), we finally obtain the following result.

Theorem 4.1. Problem (4.1), with periodic boundary conditions, has a global attractor whose fractal dimension satisfies the following two-sided estimate:

$$
2\left[\frac{L}{2 \pi}\left(\frac{\beta}{2 \alpha}+\sqrt{\frac{\beta^{2}}{4 \alpha^{2}}+\frac{\lambda}{\alpha}}\right)^{1 / 2}\right]+1 \leqslant \operatorname{dim}_{F} \mathcal{A} \leqslant 10^{1 / 4} \frac{L}{\pi}\left(\frac{\beta^{2}}{2 \alpha^{2}}+\frac{\lambda}{\alpha}\right)^{1 / 4}+\frac{5}{4} .
$$

We observe that the estimate (4.13) is sharp in terms of the physical parameters in the sense that, for all positive $\alpha, \beta$ and $\lambda$, the following inequality holds:

$$
2^{1 / 4} \geqslant\left(\frac{\beta}{2 \alpha}+\sqrt{\frac{\beta^{2}}{4 \alpha^{2}}+\frac{\lambda}{\alpha}}\right)^{1 / 2} /\left(\frac{\beta^{2}}{2 \alpha^{2}}+\frac{\lambda}{\alpha}\right)^{1 / 4} \geqslant 1
$$

Estimate (4.13) is useful in the sense that the left- and right-hand sides both show the same scalings with respect to all parameters. For example, they both scale like $\sqrt{\beta}$ for large $\beta$ and like $1 / \sqrt{\alpha}$ for small $\alpha$. In the population-dynamics context, these parameters $\beta$ and $\alpha$ measure short-range activation and long-range inhibition, respectively, and, following our remarks in $\S 1$, we may anticipate the onset of nontrivial dynamics if $\beta$ is large and $\alpha$ relatively small. Estimate (4.13) both confirms this and will be a sharp estimate for $\operatorname{dim}_{F} \mathcal{A}$ in the sense described above.

The bounds for the global Lyapunov exponents that we have used in this analysis have been obtained by classical methods. It is already quite well known (Temam 1997) that for many equations the Hausdorff dimension is bounded above by the root of a certain equation $(g(m)=0$ in this paper). It is important to stress that in this paper our bounds concern the fractal dimension. It is a corollary of what we have done that, for our particular equation, the fractal dimension admits the same upper bound as the Hausdorff dimension (the bound being the root $m=m_{0}$ of the equation $g(m)=0$ ). Furthermore, all our estimates are given in an explicit form.

\section{Concluding remarks}

In this paper we have illustrated one possible approach by which the positivity of solutions of a fourth-order reaction-diffusion problem can be studied, and we have 
presented certain conditions on the parameters and on the initial data that will ensure that positivity of solutions holds for all time.

The condition $\alpha>\frac{1}{4}$ appears in some of our theorems. This condition is necessary for our particular approach to work, but there is, we feel, no reason to suspect that the number $\frac{1}{4}$ is, in any sense, a critical value for $\alpha$. Indeed, a linearized analysis about the $u=1$ solution of (1.2) suggests that $\frac{1}{8}$ is a critical value for $\alpha$ as far as the structure of the solution set of (1.2) is concerned. Other authors who have studied an equation like (1.2), but with the $u_{x x}$ term having a positive coefficient (see, for example, Peletier \& Troy 1997), have also noted that the structure of the solution set seems to depend on the coefficient of the fourth-order spatial derivative, with $\frac{1}{8}$ being a critical value. Our analysis in the present paper apparently cannot be improved to identify $\frac{1}{8}$ as a critical value for $\alpha$.

We now discuss the role of the domain length $L$. Recall that the conditions for positivity stated in theorem 3.1 include the requirement that the initial data for (1.2) should satisfy

$$
\int_{0}^{L}(u(x, 0)-1)^{2} \mathrm{~d} x \leqslant J^{*}
$$

where $J^{*}$ is the smallest positive root of the equation $f(J)=0$, with $f(J)$ defined in the statement of theorem 2.1. If $\alpha$ is fixed and $J^{*}$ is computed numerically for various $L$, one finds that $J^{*}$ increases with $L$, but very slowly for large $L$. Numerical experimentation also suggests that $J^{*} / L \rightarrow 0$ as $L \rightarrow \infty$. Now, of course, both the right- and the left-hand sides of (5.1) involve $L$. To assess the chances of (5.1) being satisfied, it is perhaps more reasonable to divide both sides by $L$, so that the lefthand side of the resulting inequality can be interpreted as the mean of $(u(x, 0)-1)^{2}$ and the right-hand side will be $J^{*} / L$, which gets smaller as $L$ is increased. In this sense, one sees that (5.1) is a more stringent condition on the initial data for large domains than for small ones. In a similar way, one can see that the condition on $\int_{0}^{L}\left(u_{x}(x, 0)\right)^{2} \mathrm{~d} x$ in theorem 3.1 is more stringent for large domains than small ones. On the other hand, our remarks just before the statement of theorem 3.1 indicate that condition (3.5) is relatively easily satisfied.

In another study of the related problem with a positive $u_{x x}$ term, Peletier et al. (1995) studied the stationary solutions and, in particular, periodic stationary solutions. They, too, were interested in the influence on the solution set of the length of the spatial domain. Their theorem 2.7 shows that the larger the domain length $L$, the larger is the number of distinct periodic solutions that can be found by their construction (which also yields the number of zeros in $(0, L)$ of each periodic solution found). Of course, those solutions with zeros are not positive solutions. The appearance of more such solutions with increasing $L$ is in a sense consistent with the remarks of the previous paragraph, suggesting that the more we increase $L$, the smaller will be the set of initial data satisfying the hypotheses of our positivity theorem.

Finally, we mention that, although our analysis deals only with the nonlinearity $u\left(1-u^{2}\right)$, it is also possible to apply our ideas to other nonlinearities containing only odd powers of $u$ (in particular, the case $u\left(1-u^{2 n}\right.$ ) for positive integer $n$ ). We have concentrated on the case $u\left(1-u^{2}\right)$, which seems to be the most interesting and relevant case physically and biologically.

Proc. R. Soc. Lond. A (2002) 
We are very grateful to the referees for their valuable suggestions. A.A.I. acknowledges financial support from the Russian Foundation for Fundamental Research (grant nos 97-01-00541 and 9901-01157) and a grant from the University of Surrey.

\section{Appendix A.}

In Ilyin (1998), the following inequality was proved,

$$
\|u\|_{\infty} \leqslant c(l) J_{0}(u)^{(2 l-1) / 4 l} J_{l}(u)^{1 / 4 l}, \quad l>\frac{1}{2},
$$

where

$$
c(l)=\left(\frac{1}{(2 l-1)^{(2 l-1) / 2 l} \sin (\pi / 2 l)}\right)^{1 / 2},
$$

and $u$ is a periodic function on $[0, L]$ with zero average,

$$
\int_{0}^{L} u(x) \mathrm{d} x=0
$$

It was also shown in Ilyin (1998) that the constant $c(l)$ in (A 1) is sharp and there are no non-trivial extremal functions for which inequality (A 1) becomes an equality.

Suppose now that $\int_{0}^{L} u(x) \mathrm{d} x \neq 0$ and $u(x)=\bar{u}+u^{\prime}(x)$, where $\bar{u}=$ const. $\neq 0$ and $\int_{0}^{L} u^{\prime}(x) \mathrm{d} x=0$. Then, using (A 1$)$ and the inequality

$$
|\bar{u}|=L^{-1}\left|\int_{0}^{L} u(x) \mathrm{d} x\right| \leqslant L^{-1 / 2}\|u\|
$$

we obtain

$$
\|u\|_{\infty} \leqslant|\bar{u}|+\left\|u^{\prime}\right\|_{\infty} \leqslant L^{-1 / 2} J_{0}(u)^{1 / 2}+c(l) J_{0}(u)^{(2 l-1) / 4 l} J_{l}(u)^{1 / 4 l} .
$$

By substituting $u=1$ in (A 4 ), we see that the constant $L^{-1 / 2}$ is sharp.

To see that the constant $c(l)$ in (A 4$)$ is sharp, we set $L=2 \pi$ and consider the sequence

$$
u_{\mu}(x)=\mu^{2 l-1} \sum_{n=1}^{\infty} \frac{\cos n x}{\mu^{2 l}+n^{2 l}}
$$

and calculate all the three norms of $u_{\mu}$ as $\mu \rightarrow \infty$. We consider the case $l=1$, the general case being completely analogous. For

$$
u_{\mu}(x)=\mu \sum_{n=1}^{\infty} \frac{\cos n x}{\mu^{2}+n^{2}}
$$

we have

$$
\left\|u_{\mu}\right\|_{\infty}=u_{\mu}(0)=\mu \sum_{n=1}^{\infty} \frac{1}{\mu^{2}+n^{2}}=\frac{1}{\mu} \sum_{n=1}^{\infty} \frac{1}{1+(n / \mu)^{2}} \rightarrow \int_{0}^{\infty} \frac{\mathrm{d} x}{1+x^{2}}=\frac{1}{2} \pi .
$$

We calculate $J_{0}\left(u_{\mu}\right)$ and $J_{1}\left(u_{\mu}\right)$ similarly. We have

$$
\mu J_{0}\left(u_{\mu}\right)=\mu^{3} \pi \sum_{n=1}^{\infty} \frac{1}{\left(\mu^{2}+n^{2}\right)^{2}}=\frac{1}{\mu} \sum_{n=1}^{\infty} \frac{1}{\left(1+(n / \mu)^{2}\right)^{2}} \rightarrow \pi \int_{0}^{\infty} \frac{\mathrm{d} x}{\left(1+x^{2}\right)^{2}}=\frac{1}{4} \pi^{2}
$$


and

$$
\mu^{-1} J_{1}\left(u_{\mu}\right)=\mu \pi \sum_{n=1}^{\infty} \frac{n^{2}}{\left(\mu^{2}+n^{2}\right)^{2}}=\frac{1}{\mu} \sum_{n=1}^{\infty} \frac{(n / \mu)^{2}}{\left(1+(n / \mu)^{2}\right)^{2}} \rightarrow \pi \int_{0}^{\infty} \frac{x^{2} \mathrm{~d} x}{\left(1+x^{2}\right)^{2}}=\frac{1}{4} \pi^{2}
$$

as $\mu \rightarrow \infty$. If we substitute asymptotic equalities (A 5), (A 6) and (A 7) into inequality (A 4), then we see that the constant $c(1)=1$ in (A 4) is sharp.

\section{References}

Babin, A. V. \& Vishik, M. I. 1989 Attractors of evolution equations. Moscow: Nauka.

Bartuccelli, M. V., Doering, C. R., Gibbon, J. D. \& Malham, S. J. A. 1993 Length scales in solutions of the Navier-Stokes equations. Nonlinearity 6, 549-568.

Bartuccelli, M. V., Gourley, S. A. \& Woolcock, C. J. 1998a Conditions for positivity of solutions of a class of dissipative partial differential equations. Appl. Math. Lett. 11, 71-74.

Bartuccelli, M. V., Gourley, S. A. \& Woolcock, C. J. $1998 b$ Length scales in solutions of a generalized diffusion model. Physica Scr. 57, 9-19.

Bernis, F. \& Friedman, A. 1990 Higher order nonlinear degenerate parabolic equations. J. Diff. Eqns 83, 179-206.

Bleher, P. M., Lebowitz, J. L. \& Speer, E. R. 1994 Existence and positivity of solutions of a fourth-order nonlinear PDE describing interface fluctuations. Commun. Pure Appl. Math. 47, 923-942.

Chepyzhov, V. V. \& Ilyin, A. A. 2001 A note on the fractal dimension of attractors of dissipative dynamical systems Nonlin. Analysis 44, 811-819.

Cohen, D. S. \& Murray, J. D. 1981 A generalized diffusion model for growth and dispersal in a population. J. Math. Biol. 12, 237-249.

Coleman, B. D., Marcus, M. \& Mizel, V. J. 1992 On the thermodynamics of periodic phases. Arch. Ration. Mech. Analysis 117, 321-347.

Constantin, P. \& Foias, C. 1985 Global Lyapunov exponents, Kaplan-Yorke formulas and the dimension of the attractors for 2D Navier-Stokes equations. Commun. Pure Appl. Math. 38, $1-27$.

Constantin, P., Foias, C. \& Temam, R. 1988 On the dimension of the attractors in twodimensional turbulence. Physica D 30, 284-296.

Dal Passo, R., Garcke, H. \& Grun, G. 1988 On a fourth-order degenerate parabolic equation: global entropy estimates, existence and qualitative behaviour of solutions. SIAM J. Math. Analysis 29, 321-342.

Grindrod, P. 1991 Patterns and waves: the theory and applications of reaction-diffusion equations. Oxford Applied Mathematics and Computing Science Series. New York: Clarendon Press/Oxford University Press.

Ilyin, A. A. 1998 Best constants in multiplicative inequalities for sup-norms. J. Lond. Math. Soc. 58, 84-96.

Ilyin, A. A. \& Chepyzhov, V. V. 2000 On the fractal dimension of invariant sets; applications to Navier-Stokes equations. Keldysh Institute of Applied Mathematics, Russian Academy of Science. Preprint 22. (In Russian).

Kalies, W. D. \& van der Vorst, R. C. A. M. 1996 Multitransition homoclinic and heteroclinic solutions of the extended Fisher-Kolmogorov equation. J. Diff. Eqns 131, 209-228.

Kalies, W. D., Kwapisz, J., van den Berg, J. B. \& van der Vorst, R. C. A. M. 2000 Homotopy classes for stable periodic and chaotic patterns in fourth-order Hamiltonian systems. Commun. Math. Phys. 214, 573-592.

Proc. R. Soc. Lond. A (2002) 
Leizarowitz, A. \& Mizel, V. J. 1989 One dimensional infinite-horizon variational problems arising in continuum mechanics. Arch. Ration. Mech. Analysis 106, 161-194.

Marcus, M. 1993 Uniform estimates for a variational problem with small parameters. Arch. Ration. Mech. Analysis 124, 67-98.

Peletier, L. A. \& Troy, W. C. 1996 Chaotic spatial patterns described by the extended FisherKolmogorov equation. J. Diff. Eqns 129, 458-508.

Peletier, L. A. \& Troy, W. C. 1997 Spatial patterns described by the extended FisherKolmogorov equation: periodic solutions. SIAM J. Math. Analysis 28, 1317-1353.

Peletier, L. A. \& Troy, W. C. 1998 Multibump periodic travelling waves in suspension bridges. Proc. R. Soc. Edinb. A 128, 631-659.

Peletier, L. A., Troy, W. C. \& van der Vorst, R. C. A. M. 1995 Stationary solutions of a fourthorder nonlinear diffusion equation. Diff. Eqns 31, 301-314.

Pomeau, Y. \& Manneville, P. 1980 Wavelength selection in cellular flows. Phys. Lett. A 75, 296-298.

Protter, M. H. \& Weinberger, H. F. 1984 Maximum principles in differential equations. Springer.

Swift, J. B. \& Hohenberg, P. C. 1977 Hydrodynamic fluctuations at the convective instability. Phys. Rev. A 15, 319-328.

Temam, R. 1997 Infinite dimensional dynamical systems in mechanics and physics, 2nd edn. Springer.

van den Berg, J. B. 2000 The phase-plane picture for a class of fourth-order conservative differential equations. J. Diff. Eqns 161, 110-153. 